\title{
La evaluación y su incidencia en la deserción escolar: ¿Falla de un sistema, de las instituciones educativas, del docente 0 del estudiante?*
}

\author{
Luz Marina Silvera Fonseca ${ }^{1}$ \\ orcid.org/0000-0001-9567-3862 \\ Universidad Libre Seccional Barranquilla, Colombia
}

DOI: http://dx.doi.org/10.17081/eduhum.18.31.1381

Recibido: 18 de marzo de 2016

Aceptado: 30 de agosto de 2016

\section{Evaluation and incidence of school desertion: a system's failure caused by the institution, the teacher or the student?}

\begin{abstract}
Palabras clave:
Evaluación,

Educación instrumental,

Deserción,

Desempeño académico.
\end{abstract}

Keywords:

Evaluation,

Instrumental education,

Desertion, Academic performance.

\begin{abstract}
Resumen
El problema de la evaluación asociada a la deserción en instituciones educativas merece una atenta reflexión como la produce este artículo que luego de una revisión bibliográfica fija el objetivo de realizar un análisis sobre el tema en el contexto particular de la educación básica. En general, se concluye que a pesar de muchos esfuerzos por minimizar los efectos de una forma de evaluación instrumental, esta todavía prevalece a sabiendas de las consecuencias negativas que trae para algunos estudiantes y para el mismo sistema educativo. Lo cierto es que aún no hemos superado la visión técnica que sobre la evaluación se tiene, por cuanto el examen y control como sinónimos de medición del desempeño están afectando la permanencia escolar.
\end{abstract}

\begin{abstract}
The problem of evaluation associated to desertion in educational institutions deserves a thoughtful analysis, just as it has been tackled in this article that after a bibliographical review focuses the aim on an analysis about the particular context of basic education. In general, it is concluded that in spite of making so many efforts to minimize the effects of an instrumental evaluation, this still prevails even after knowing about the negative consequences it brings to students and to the educational system. The only certain fact is that we have not yet overcome the vision about evaluation, for as far as exam and controls are concerned, they are synonyms for measuring performance, and this is affecting school permanence.
\end{abstract}

\section{(c) $\$$}

Referencia de este artículo (APA): Silvera, L. M. (2016). La evaluación y su incidencia en la deserción escolar: ¿Falla de un sistema, de las instituciones educativas, del docente o del estudiante? Revista Educación y Humanismo, 18(31), 313-325. http://dx.doi.org/10.17081/eduhum.18.31.1381

\footnotetext{
* Este artículo de reflexión está asociado al proyecto: Factores asociados a la deserción escolar en la educación básica y media de los colegios oficiales del distrito de Barranquilla y del departamento del Atlántico.

1. Doctorante en Educación. Magíster en Administración, Trabajadora Social y Contadora Pública. Profesora tiempo completo de la Universidad Libre Seccional Barranquilla. Email: 1silvera@unilibrebaq.edu.co
} 


\section{Introducción}

La evaluación del aprendizaje por lo general supone un juicio de valor, cuyos procedimientos de comparación entre objetivos de enseñanza y resultados llevan al docente a buscar una información que asegure de modo imparcial y objetivamente el reporte de los desarrollos de los estudiantes. Por ello como su meta principal es determinar el valor de algo, en este caso del aprendizaje que está siendo enjuiciado por el docente, asume interpretaciones radicales y en ocasiones subjetivas, que amparadas en el poder de los evaluadores en algunas ocasiones impiden develar los verdaderos obstáculos del progreso académico del estudiante.

Desde esta perspectiva se abordarán los problemas de la evaluación en el nivel de la educación básica, comenzando por reflexionar sobre los intereses que se mueven alrededor de la evaluación en tanto inciden en el logro o no de los resultados del aprendizaje de los estudiantes y sobre las consecuencias que esta valoración pueda tener sobre los contextos culturales, sociales, económicos, familiares y psicológicos en los cuales el estudiante se encuentra inmerso. Entre estas consecuencias se inscriben la deserción y el fracaso escolar que se vienen presentando en algunas instituciones educativas de Colombia.

En realidad, alrededor de la evaluación se ha tenido un marcado interés técnico o instrumental, asociado al mundo de la medición, la competitividad y la normativa, que mantiene tanto a las instituciones educativas como a los actores académicos (estudiantes, docentes, padres de familia), y al mismo sistema educativo, en un sueño profundo. Es el sueño profundo de los resultados que en ocasiones se convierte en la enfermedad de la evaluación. Esta práctica pedagógica evaluativa de tipo técnica es lo que Habermas (1971, citado en Grundy, 1998) 1lama acción instrumental y acción estratégica. La primera, regida por reglas técnicas basadas en el conocimiento empírico, implica predicciones que pueden resultar correctas o incorrectas. La segunda, depende solo de la correcta evaluación de posibles elecciones alternativas, resultante del cálculo, complementado por valores y máximas. Desde aquí se confía en la predicción de los resultados gracias a los objetivos que determinan los estándares.

"Cuando predomina el interés técnico, la evaluación supone valorar la eficacia del curriculum en relación con la medida en que el producto $<<$ se ajusta $>>$ al eidos orientador" (Stenhouse, 1975, p.121). Recordemos que el interés técnico refleja, ante todo, un interés por el control. No solo controla la puesta en marcha del currículo, así mismo controla a los alumnos de modo que puedan llegar hasta donde los diseñadores del contenido curricular han planeado. Dicho principio, que se transpola al proceso de evaluación, consiste en la necesidad de efectuar una valoración del producto en la medida en que este se ajusta a los objetivos que guía su preparación. Se valora el producto, y la evaluación, para que goce de autoridad y legitimidad. 
Así, el currículo se entiende como un plan de instrucciones o mero instrumento orientado al producto sobre propósitos determinados y preestablecidos para conseguir un fin, cuyo centro son los resultados de aprendizaje esperados en los estudiantes, y en donde el maestro es un técnico que lo pone a funcionar para conseguir los objetivos fijados, por lo que su papel es esencialmente mecanicista. Como bien señala Grundy (1998), este interés da lugar a una determinada forma de acción orientada a productos, durante la cual se reproducen planes pensados y elaborados por otros, y que se materializa en la práctica educativa. Se trata de una acción instrumental maniobrada por un conjunto de normas técnicas orientadas hacia el control de la realidad. En resumen, el interés técnico constituye un asunto fundamental por el control del ambiente mediante la acción, de acuerdo con reglas basadas en leyes con fundamento empírico. Tal interés ha llevado a reducir la evaluación a la simple medición del rendimiento académico, orientada por el control del medio para obtener unos resultados que generalmente define el docente. Esto lleva a enfatizar el control del aprendizaje, apegado no solo a unos objetivos y reglas sino a contenidos delimitados previamente (Tyler, 1986).

A pesar de los avances obtenidos en materia de evaluación, seguimos atrapados por un sistema tradicional de valoración que supone unas calificaciones sobre pruebas de conocimiento e información, pues aunque hoy día se llamen integrales y cualitativas, privilegian estándares centrados en procesos básicos de pensamiento que van describiendo los logros y dificultades de los estudiantes a través de escalas cualitativas, que de suyo siguen siendo cuantitativas. En consecuencia, por lo general, la evaluación es unificada y unificadora para todos los estudiantes. Por ello esta experiencia se convierte en fuente de tensiones, contradicciones y frustraciones, dada la visión examinadora de control y rigidez que mantienen, ya que por lo general el estudiante deberá enfrentarse a sus pares a fin de demostrarle al docente en qué punto del ranking se encuentra.

De esta forma la evaluación técnica viene marcada por una concepción muy particular del docente, quien asume un papel autoritario y vertical que ha venido consolidando, entre otras cosas, a través de las experiencias vividas previamente en su etapa estudiantil. De cierta manera, los evaluadores, a su vez, consciente o inconscientemente reflejan o proyectan en las prácticas que siguen con sus estudiantes la forma como fueron evaluados.

Frente a esta tendencia, Grundy (1998), siguiendo a Habermas (1971), plantea un currículo práctico, el cual valida la categoría de los alumnos como sujetos del aprendizaje, y no como objetos, durante un acontecimiento curricular que se ajusta a los intereses de los objetivos. Esto supone que la preocupación fundamental del profesor será el aprendizaje, no la enseñanza, lo que lleva a que los procesos de evaluación cifren su interés tanto en la acción e interacción como en el resultado, por lo que se deduce que carece de 
sentido hablar de la evaluación de la eficacia del currículo en términos de objetivos especificados de antemano (Grundy, 1998). Desde un interés práctico la evaluación se convierte también en una parte integrante del proceso educativo en su conjunto y no se queda algo separado. Stenhouse (1975, p.121, citado en Grundy, 1998) manifiesta: "Deseo argumentar contra la separación entre el encargado de desarrollar (el curriculum) y el evaluador, y en favor de una investigación integrada del curriculum". Si el interés básico es práctico, la evaluación significará elaborar juicios acerca de la medida en que el proceso y las prácticas desarrolladas a través de la experiencia de aprendizaje favorecen el $<<$ bien $>>$ de los participantes.

Al respecto (Grundy, 1998, p.109) dice: “el interés cognitivo práctico significa que el contenido curricular estará determinado por consideraciones sobre el $<<$ bien $>>$ en vez de por lo que se debe seleccionar para su enseñanza a fin de lograr un conjunto de objetivos especificados de antemano"; dado que, en cualquier selección de contenidos, la importancia reside en la construcción del significado y en la interpretación. En este sentido, el aprendizaje supone la construcción de significados. De esta manera el currículo, en específico: la evaluación, estimulará la interpretación, comprensión y el ejercicio del juicio tanto del estudiante como del docente, dejando a un lado el aprendizaje rutinario con conceptos preestablecidos.

Los procesos evaluativos vistos a partir de lo que es el paradigma emancipador o sociocrítico plantean que el aprendizaje es reconocido como un acto social. Refiere Grundy (1998) que "la praxis supone un proceso de construcción del significado que reconoce a este como construcción social" (p.161). La praxis es de carácter integrador ya que se desarrolla en el mundo de la interacción: el mundo social y cultural; así, mediante el acto del aprendizaje los alumnos son participantes activos en la construcción de su propio conocimiento. Es más, esa construcción estaría determinada por intereses humanos fundamentales que suponen conceptos de personas y de su mundo.

Gran parte de lo expresado anteriormente evidencia que el interés emancipador supone que la evaluación no puede tratarse como un aspecto separado del proceso de construcción del currículo. Para empezar, un interés emancipador significa cesar la opresión de la evaluación externa del trabajo de los prácticos. Los procesos de evaluación informados por un interés emancipador no son azarosos ni restrictivos ni mecánicos, tampoco son punitivos sino que posibilitan el desarrollo de un pensamiento crítico, una acción autorreflexiva, responsable, autónoma para tomar las riendas de su propia vida, de tal manera que la evaluación estará relacionada recíprocamente integrada en el proceso.

Al respecto Grundy (1998, p.142) señala que en el interés técnico procedimental e instrumental se le atribuye al discente el rol de receptor pasivo del acto educativo; inverso a este, el interés 
emancipador involucra y/o hace parte integral al estudiante del acto educativo, no solo como receptor activo del saber, sino como constructor de su propio conocimiento en compañía del profesor.

Para Habermas (1972, pp.205 y ss.), emancipación significa "independencia de todo lo que está fuera del individuo, y se trata de un estado de autonomía más que de libertinaje".

Cuando la sociedad en la que nos movemos orienta sus dinámicas culturales, económicas, políticas y sociales hacia los resultados, la evaluación técnica se generaliza. Miremos, por ejemplo, los principales indicadores asociados a la calidad de la educación. Tanto en Colombia como en otros países estos se centran en las pruebas estandarizadas que se convierten en el insumo para la elaboración de los rankings de las instituciones educativas, y que van afianzando cada vez más el interés técnico. Las instituciones educativas han demostrado mayor interés en responder y atender las necesidades de información de los resultados de sus alumnos que en mejorar sus procesos evaluativos y ayudar a sus docentes y estudiantes a fortalecer sus métodos de evaluación tradicional que han utilizados durante mucho tiempo.

Nuestro sistema de educación presiona $\mathrm{y}$ arrastra a las instituciones educativas a estar en una constante de-formación por objetivos y resultados sin importar las consecuencias que esto traiga para los alumnos y su familia. Es en este panorama donde se convierte la evaluación en el verdugo que define si el estudiante obtuvo o no los logros esperados en su proceso de aprendizaje, y que en ocasiones se lleva por delante los sueños personales, las aspiraciones de una mejor condición de vida. En ese contexto, la presión por los buenos resultados puede generar algunos problemas como la deserción o el fracaso académico o repitencias en los niveles de educación básica.

Desde las instancias administrativas se les confiere a las instituciones educativas la responsabilidad social de desarrollar e implementar su sistema interno de evaluación y promoción de los estudiantes que dé cuenta de la calidad de la educación colombiana. No obstante, sistemas de evaluación cuantitativos centrados en la calificación y la medición presionan a los docentes para el logro de resultados de aprendizaje; a su vez, estos presionan a los padres de familia para que continúen el control de sus hijos por el rendimiento, y, al final, sobre los estudiantes recae toda la responsabilidad de avanzar y obtener unos buenos resultados. Con este impulso, algunos de ellos desertan o fracasan académicamente.

La deserción es un problema de política educativa y está relacionada con procesos de calidad. Si bien existen múltiples perspectivas en la conceptualización de la calidad, hay consenso en cuanto a que ella se articula con la evaluación y la innovación, así como sobre la necesidad de reflexionar acerca de tres factores claves 
del sistema educativo: la pertinencia, la eficacia y la eficiencia. El primero de estos elementos, es decir, la pertinencia, se define como la coherencia entre el proyecto educativo institucional y sus programas específicos con las exigencias del entorno. El segundo, la eficacia, se relaciona con la coherencia entre acciones para el logro de las metas de la institución y tercero, la eficiencia, asocia la coherencia entre los recursos invertidos, el esfuerzo desplegado y el tiempo empleado para el logro de los objetivos del programa y los fines de la institución. Estos factores son parte integral del análisis al que deben someterse las políticas educativas que definen las estrategias para enfrentar la deserción.

Aunque el fracaso escolar obedece a diversos factores, es innegable que por lo menos inciden en él de manera directa, el maestro (enseñanza), el alumno (aprendizaje) y la evaluación como procesador articulador y legitimador de dicho proceso. Díaz Sánchez (1999) afirma que los estudiantes se mueven entre la aceptación y el rechazo, como sujetos de procesos de evaluación y de control disciplinario, donde se encuentran y desencuentran sus visiones y valores particulares con las de los maestros, lo que hace pensar de manera diferente el fracaso escolar y la práctica técnica del docente.

El Ministerio de Educación Nacional colombiano (MEN, 2014) define la deserción escolar como la interrupción o desvinculación de los estudiantes del sistema educativo. Es decir, que niños y jóvenes que asisten al colegio dejan de hacerlo y no logran culminar sus estudios e identifica dos tipos de deserción, según su duración: temporal o definitiva. Algunos niños que abandonan algún curso pueden matricularse al año siguiente (deserción temporal), mientras que en otros casos los estudiantes que dejan el sistema educativo no retornan.

\section{Antecedentes}

En sus investigaciones, Jara, Velarde, Gordillo, Guerra, León, Arroyo y Figueroa (2008) manifiestan que las causas más específicas del bajo rendimiento académico son las dificultades que en cuanto a capacidad de autoaprendizaje y aprendizaje presentan los estudiantes. Ellos plantean que sigue predominando el aprendizaje memorístico en los alumnos que no permite el desarrollo de capacidades de reflexión, crítica, análisis y síntesis propias de metodologías tradicionales de enseñanza cuya evaluación convencional sigue centrada en los exámenes y restringida en los resultados. Sus investigaciones concluyen que los alumnos universitarios con rendimiento académico bajo que no lleguen a resolver sus problemas son los más propensos a presentar mayor grado de deserción, desmotivación, ausentismo, repetición. Esto coincide con lo planteado por Merlino, Ayllón y Escanés (2011), quienes afirman que la incorrecta aplicación de la evaluación sumativa impide observar mejores resultados del rendimiento, así como un mejor nivel en los educandos, ya que existe una relación entre el rendimiento académico y el índice de riesgo de abandono escolar definitivo dadas las normas de evaluación del aprendizaje preestablecidas. 
La aplicación de una evaluación inadecuada de los aprendizajes por parte de los docentes puede incidir en el rendimiento académico; por lo tanto, los maestros tienen un rol muy importante. Al respecto Moreno (2009) afirma que si bien se han hecho muchos esfuerzos por desmarcar un currículo rígido y enciclopédico, incluso moviéndose hacia modelos flexibles que enfaticen un paradigma centrado en el aprendizaje del alumno, la evaluación se ha mantenido prácticamente intacta, con metodologías cuantitativas.

En sus investigaciones Moreno (2009) precisa que aunque hoy en día contamos con enfoques de enseñanza y de aprendizaje más amplios e integrales, la rigidez de la evaluación sigue centrada en los exámenes y restringida a los resultados de los alumnos, negándose a ceder su paso a una evaluación formativa y continua, lo que evidencia que las tendencias teóricas y metodológicas actuales van por un sendero y las prácticas pedagógicas por otro muy distinto (p.587).

Se observa cómo persiste una evaluación convencional que condiciona y subordina fuertemente todo el proceso: las pruebas tradicionales constituyen la técnica de evaluación por excelencia y el rol del maestro en el aula refiere a un controlador del comportamiento del alumno que asocia el estudio como actor un pasivo ajustable a los intereses de los objetivos, y al que se le responsabiliza de su fracaso escolar.

Pérez (2013), Quiñónez y Barraza (2013), Villena (2013), Ortega y Ramírez (2013) con- cuerdan en que la evaluación sumativa equivocada incide en el rendimiento académico. Esta forma de evaluación articulada en metodologías conductistas se encuentra distante del enfoque de competencias que actualmente se promueve. Dejan ver la necesidad de reconceptualizar los sistemas institucionales de evaluación, repitencia escolar y promoción, y de mirar hacia nuevas prácticas pedagógicas que cumplan el verdadero propósito de mejorar los aprendizajes en los alumnos. El análisis crítico sobre la evaluación permitirá comprender el fracaso escolar como una respuesta distinta a las exigencias de sumisión, sujeción y obligatoriedad a las normas preestablecidas, por lo que no se estaría hablando de un proceso individual sino más bien de un proceso social, que permite ver el fracaso escolar como un proceso de resistencia ante una realidad reproductora, limitante, que enfatiza en indicadores numéricos más que en logros académicos. El rechazo a esto hace que los alumnos presenten dificultades que pueden llevarlos a desertar del sistema escolar. Algunos autores como Acosta (2009), Zárate y Socha (2009) coinciden en sus investigaciones cuando afirman que el fenómeno de la deserción escolar se encuentra estrechamente asociado y relacionado con causas académicas y en aspectos relacionados con el aprendizaje tales como políticas de evaluación, la evaluación, los métodos y metodologías utilizados por las instituciones educativas y respaldadas por el mismo Estado, que inciden significativamente sobre la decisión de desertar temporal o definitiva del sistema educativo. 
Loyola, Espinosa-Díaz, González, Santa Cruz-Grau y Castillo-Guajardo (2014) en su investigación concluyen que por lo general quien deserta lo hace por problemas académicos sin que haya recibido con anterioridad ayuda ni de su familia ni de la escuela; la mayoría de los desertores posee un trayecto escolar caracterizado por repeticiones de curso, mal rendimiento académico, mal comportamiento, ausencias frecuentes a clases y sucesivos cambios de escuela. Estas situaciones constatan una débil capacidad de las familias y de los propios establecimientos para enfrentar las dificultades. Como se ha observado en el caso de muchos de los desertores, la respuesta de la institución escolar suele ser la expulsión o la negación de matrícula al alumno considerado de difícil enseñanza.

La búsqueda bibliográfica permitió identificar investigaciones realizadas en México, Chile, Argentina, Ecuador y Colombia que abordan la relación entre los problemas de la evaluación del aprendizaje y los altos índices de deserción escolar en Latinoamérica. En el contexto colombiano son escasas las investigaciones y/o estudios que sobre este problema se han hecho a nivel de educación básica, entendiéndola como básica primaria y básica secundaria.

Precisamente, en su investigación, Salvámut, Oliver y Comas (2014) aseguran que el fenómeno de la deserción debe afrontarse desde la multidimensionalidad de los factores que la originan, y que los autores entienden como producto de la combinación, mezcla y correlación de aquellos; esto quiere decir que el asunto debe ser estudiado como parte de un proceso, no como un hecho puntual y aislado, sino como el resultado del alejamiento creciente de la escuela. Estos aspectos juntos dan una aproximación integradora.

\section{El caso colombiano}

Si bien las estadísticas muestran que la deserción en Colombia disminuyó de manera sistemática durante el periodo 2003-2011, la reducción de dichos porcentajes no es tan contundente como se esperaría. En el 2003 se reportó un 7,5 \% de deserción (Muñoz, 2013); en el 2009 la población que abandonó el sistema educativo fue de 409.275, equivalente al 5,15\%; en el 2010 la población desertora representó el 4,89 \% y para el 2011 la población que desertó fue de 360.780 equivalente al 4,53 \%. Muñoz (2013) y Ramírez y Ramírez (2014) afirman que Colombia ha tratado de avanzar en estrategias que disminuyan la deserción y la repitencia escolar a fin de evitar así que más de 490 mil estudiantes abandonen sus estudios. Pero aún falta más por hacer. El propósito plasmado en el Plan Nacional de Desarrollo es lograr reducir la deserción a 3,8 \% en 2014, es decir, evitar la deserción de otros 50 mil estudiantes en los años siguientes (Ministerio de Educación Nacional [MEN]-SIMPADE, 2014).

Ante más de 30 causas de deserción identificadas por el Ministerio de Educación Nacional a través de la Encuesta Nacional de Deserción (ENDE 2009-2010), y que tienen como factores asociados las dinámicas de las instituciones educativas, llama la atención la forma como se repiten en los departamentos de Atlántico, Sucre, Bolívar, La Guajira, Quindío, Santander y 
Vaupés los problemas relacionados con las estrategias pedagógicas inadecuadas asociadas a la evaluación y las dificultades académicas, por lo que resulta importante ponerles atención si lo que se desea es minimizar su efecto sobre la deserción escolar.

A nivel local, en Barranquilla, la tercera ciudad en importancia en Colombia, según la Secretaría de Educación Distrital (2012) en su Boletín Estadístico, se presenta una tasa de deserción del $4.486 \%$, lo que representa un total de 9.212 estudiantes. Al comparar esta tasa frente a la del año inmediatamente anterior se evidencia un aumento de $0,86 \%$, pues se pasa del $3.670 \%$ en el año 2010 a $4.486 \%$ en el año 2011, con lo cual se demuestra la existencia de ciertas fallas en la implementación de estrategias de acceso y permanencia.

Adicionalmente, la tasa de reprobación o repitencia en las instituciones educativas del distrito de Barranquilla aumentó en un 5,0\%, tomando como referencia el año 2010, en donde se reportaron 14.489 estudiantes, cifra que concentra este problema en los niveles de primaria y secundaria. Cabe anotar que la tasa de repitencia corresponde a la relación existente entre los estudiantes que se reportan como repitentes (estudiantes que permanecen en un mismo grado escolar durante un periodo mayor a un año), con respecto a los alumnos matriculados en ese mismo grado o nivel.

Tinoco, Herrera y Acosta (2008) y Enríquez, Segura y Tovar (2013) en sus investigaciones muestran que el bajo rendimiento académico es una de las causales de la repitencia y la deserción que influye en el fracaso escolar, y lo atribuyen a factores que tienen relación directa con las instituciones educativas, el rol docente y el sistema de evaluación utilizado por la institución educativa. Lo anterior concuerda con las apreciaciones realizadas en sus investigaciones por Ortega y Ramírez (2013); Castro y Bravo (2013), en cuanto a que son muchos los factores que influyen en el rendimiento académico de los estudiantes: desde la mirada de las instituciones educativas, de los docentes, de los padres de familia, de los estudiantes hasta del mismo sistema educativo. Por lo tanto, estos investigadores invitan a reflexionar sobre la reconceptualización del alumno como único responsable de su fracaso, así como a reconocer la validez de otras prácticas pedagógicas, $\mathrm{y}$, también, a mirar diferentes sistemas de evaluación que satisfagan de manera eficiente el propósito de la educación y el mejoramiento de los aprendizajes en los estudiantes. En este sentido, la evaluación no debe permitir que se observe al alumno como un número o un logro cuantitativo en su desempeño académico. Al respecto Garzón (2012) y Chaverra y Gómez (2013) en sus planteamientos señalan que la falla en el contexto educativo lleva a que los estudiantes tomen la decisión de desertar, con lo cual establecen una clara relación entre la evaluación y su determinación de salir del sistema. Los autores afirman que aun cuando se ha hecho un sinnúmero de esfuerzos por transformar estas prácticas tradicionales en evaluaciones formativas, esto no ha sido fácil, y ello influye en las concepciones que al respecto adoptan los 
alumnos sobre la evaluación; tanto es así que los estudiantes tienen como percepción que la evaluación realizada por sus docentes es infalible y válida para medir, valorar y calificar sus conocimientos, razón por la cual buscan obtener una nota o calificación a través de un proceso netamente cuantitativo. En este sentido, Cajigas y García (2014) afirman que se sigue privilegiando no solo la transmisión de contenidos alejados de la realidad de los alumnos, sino que también se sigue evaluando la repetición memorística de los contenidos preestablecidos por los docentes. Todo esto explica por qué en más de una ocasión el estudiante rechaza los estudios y toma la decisión de abandonar la institución educativa, suspendiendo su formación, y asume una actitud negativa frente al proceso de aprendizaje.

\section{Conclusiones}

Aunque en los últimos años son numerosos los esfuerzos realizados a nivel local, nacional e internacional para cambiar la concepción técnica que sobre la evaluación se tiene y trascender de lo cuantitativo y de la evaluación por objetivos a un sistema de evaluación más cualitativo, humanizante e integral del alumno, se concluye que hay necesidad de algo más que cambios en el discurso y la legislación educativa, dado que seguimos dominados y arrastrados por un sistema de evaluación estandarizado, coercitivo, punitivo, autoritario, que privilegia la comparación y el éxito (Moreno, 2009). Para Garzón (2013), la evaluación vista como un elemento represivo podría llevar al fracaso rutinario, provocando un aumento en los índices de deserción escolar y reprobación. Al retomar las investigaciones ela- boradas en los últimos cinco años Garzón (2012) observa que no se está llevando a cabo el verdadero propósito de la evaluación y cómo se está afectando el proceso de aprendizaje.

Bajo esta perspectiva, se continúa considerando la evaluación como sinónimo de medición en donde vale únicamente la nota obtenida. Así, el tipo de técnica de evaluación empleada se diseña bajo un esquema tradicional $\mathrm{y}$, por consiguiente, los instrumentos se enfocan particularmente a verificar la parte memorística, en donde la mayoría de veces y utilizan pruebas escritas (Garzón, 2012).

Según el Ministerio de Educación Nacional (MEN, 2008), teniendo en cuenta el aspecto ético de la evaluación, se hace evidente que esta tiene una implicación directa sobre el desarrollo de la personalidad y el desempeño futuro del estudiante en la sociedad, ya que se encuentra en un proceso de formación. Por tal razón la evaluación debe resignificarse no como una metodología amenazante para medir el conocimiento ni como un instrumento de castigo. Se hace evidente la preocupación de las instituciones educativas por satisfacer necesidades de información de los resultados de sus alumnos, todo ello bajo el influjo de agentes externos que basan la calidad de los aprendizajes en la posición o ranking que ocupa el estudiante o la institución; en un mundo de interés técnico prevalece el empeño en alcanzar los objetivos sin reparar lo que cueste, pues solo importa que gane el mejor según lo que indican los resultados. 
Sigue entonces predominando la prueba o el examen tradicional como la mejor técnica de evaluación por excelencia, aun cuando no siempre cumple una función pedagógica, formadora, sino, en muchos casos, la de satisfacer necesidades burocráticas y de rendición de cuentas como dice Moreno (2009) en su artículo "La evaluación del aprendizaje en la universidad: tensiones, contradicciones y desafíos".

Esta visión del llamado mundo de la evaluación técnica hace énfasis en los resultados antes que en los procesos de aprendizaje, haciendo sentir muchas veces al alumno responsable de su fracaso escolar, lo que en ocasiones influye en su deserción temporal o definitiva de las instituciones educativas y, por ende, del sistema.

Cuán grande es el daño que este sistema está causando a los alumnos, en los aspectos social, económico y emocional, por cuanto viene frustrando su vida, sus sueños y sus metas y cuán grande es el daño que el mismo sistema se hace permitiendo que así se obstaculice el desarrollo de la educación y que haya un freno que cuestiona la calidad de la educación.

La deserción y la repitencia escolar son consecuencias de esta situación y vemos cómo a nivel nacional e internacional se agotan los esfuerzos económicos, sociales, culturales y académicos por contrarrestarlas, si bien estos esfuerzos no pasan de ser paños de agua tibia, porque la realidad es que nada se ha podido controlar si cada día más alumnos desertan del sistema educativo por problemas académicos, tales como dificultades en las pedagogías de enseñanza, en las metodologías, en el rendimiento académico, en la formas de evaluar, en el proceso de enseñanza-aprendizaje centrado en la transmisión de conocimiento.

\section{Referencias}

Acosta, M. (2009). Los procesos de aprendizaje y su incidencia en la deserción estudiantil en el Programa de Química Farmacéutica de la Universidad de Cartagena en el periodo del 2003 al 2007. Psicología desde el Caribe, 24.

Cajigas, R. \& García, Y. (2014). Aprendizajes en las Ciencias Naturales, una compilación bibliográfica (2010-2013). Santiago de Cali: Universidad del Valle.

Castro, J. \& Bravo, F. (2013). Diferentes miradas acerca de las causas del desempeño bajo en los estudiantes de la institución educativa Vasco Núñez de Balboa, Cauca, Colombia. Tesis de maestría no publicada, Universidad de Manizales, Caldas, Colombia.

Chaverra, L. \& Gómez, C. (2013). Interpretando el fenómeno de la deserción escolar en la Institución Educativa Rural Granjas Infantiles del municipio de Copacabana. Medellín: Universidad de San Buenaventura Seccional Medellín.

Díaz Sánchez, J. (noviembre, 1999). La relación docente/alumno en la escuela secundaria: de la exclusión al fracaso escolar. Trabajo presentado en el V Congreso Nacional de Investigación Educativa, Aguascalientes, México. 
Enríquez, C., Segura, A. \& Tovar, J. (2013). Factores de riesgo asociados a bajo rendimiento académico en escolares de Bogotá. Revista Investigaciones Andinas, 15(26), 654-666.

Garzón, R. (2012). Concepto y finalidad de la evaluación para los estudiantes del grado quinto de primaria del Colegio San Simón, sede Restrepo, del municipio de Ibagué. Tesis de maestría no publicada, Universidad del Tolima.

Garzón, R. (2013). Concepto y finalidad de la evaluación para los estudiantes del grado quinto de primaria del Colegio San Simón, sede Restrepo, del municipio de Ibagué. Ibagué: Universidad del Tolima.

Grundy, S. (1998). Producto o praxis del curriculum ( $3^{\mathrm{a}}$ ed.). Madrid: Ediciones Morata.

Habermas, J. (1971). Conocimiento e interés. España: Edición Taurus.

Habermas, J. (1972). Conocimiento e interés. España: Edición Taurus.

Jara, D., Velarde, J., Gordillo, G., Guerra, G., León, I., Arroyo, C. \& Figueroa, M. (2008). Factores influyentes en el rendimiento académico de estudiantes del primer año de Medicina. Anales de la Facultad de Medicina, 69(3), 193-197.

Loyola, J., Espinosa-Díaz, O., González, E., Santa Cruz-Grau, E. \& Castillo-Guajardo, D. (2014). Deserción escolar en Chile: un estudio de caso en relación con factores intraescolares. Educación y Educadores, 17(1), 32-50.
Merlino, A., Ayllón, S. \& Escanés, G. (2011). Variables que influyen en la deserción de estudiantes universitarios de primer año. Construcción de índices de riesgo de abandono. Revista Electrónica Actualidades Investigativas en Educación, 11(2), 1-30. Ministerio de Educación Nacional (2008). La evaluación del aprendizaje. Tercer documento de trabajo. Diálogo Nacional Sobre la Evaluación del Aprendizaje en el Aula Plan Decenal de Educación. Bogotá: Autor.

Ministerio de Educación Nacional (MEN)-SIMPADE (2014). Sistema de Información para el Monitoreo, Prevención y Análisis de la Deserción Escolar en Educación Preescolar, Básica y Media. Convenio con la Corporación Colombia Digital-(CCD). Bogotá: Autor.

Ministerio de Educación Nacional \& Universidad Nacional de Colombia (s.f.). Encuesta Nacional de Deserción Escolar (ENDE)(2009-2011). Bogotá: Autores.

Moreno, T. (2009). La evaluación del aprendizaje en la universidad: tensiones, contradicciones y desafíos. Revista Mexicana de Investigación Educativa, 14(41), 563-591. Muñoz, C. (2013). Deserción escolar, un concepto que no concluye: casos de no conclusión satisfactoria del ciclo escolar en la institución educativa de Santa Librada. Trabajo de grado, Universidad del Valle, Santiago de Cali.

Ortega, L. \& Ramírez, J. (2013). Implicaciones del sistema institucional de evaluación en 
la reprobación de estudiantes: un estudio de caso. Recuperado desde el repositorio de la Universidad Católica de Pereira. Disponible en: http://ribuc.ucp.edu.co:8080/ jspui/bitstream/handle/10785/1774/ Art\%C3\%ADculo_5.pdf? sequence=2

Pérez, L. (2013). La evaluación sumativa y su incidencia en el rendimiento académico de los estudiantes de bachillerato de la Unidad Educativa Fiscal Mixta Dario Guevara, de la ciudad de Ambato, provincia de Tungurahua. Recuperado desde el Repositorio Digital Universidad Técnica de Ambato. Disponible: http://repositorio. uta.edu.ec/jspui/handle/123456789/4859

Quiñónez, J. \& Barraza, A. (2013). La evaluación del aprendizaje en educación media superior. Dificultades y posibles soluciones. Revista Electrónica Praxis Investigativa de la Red Durango de Investigadores Educativos A. C., 5(9), 52-58.

Ramírez, I. \& Ramírez, J. (2104). Análisis de la deserción en el sistema educativo oficial de San José de Cúcuta (Colombia). Serie Documentos de Trabajo de Economía Regional y de Frontera. Pamplona: Observatorio Socioeconómico Regional de la Frontera. Universidad de Pamplona.

Salvà-mut, F., Oliver, M. \& Comas, R. (2014, enero-junio). Abandono escolar y desvinculación de la escuela: perspectiva del alumnado. Magis, 6(13), 129-142.

Secretaría de Educación Distrital (2012). Boletín Estadístico 2012-II. Recuperado desde: http://www.sedbarranquilla.gov.
co/sed/documents/Boletin\%20Estadistico2012\%20-\%20II.pdf

Stenhouse, L. (1975). An introduction to curriculum research and development. Portsmouth: Heinemann Publishing.

Stufflebeam, D. \& Shinkfield, A. (1987). Evaluación Sistemática. Guía teórica y práctica. Barcelona-Buenos Aires-México: Ediciones Paidós.

Tinoco, R., Herrera, E. \& Acosta, A. (2008). Factores socioeconómicos asociados a la deserción escolar de los estudiantes de la media secundaria de las instituciones educativas oficiales del sector urbano del municipio de Arjona durante los años 2005-2006. Revista de Investigación y Pedagogía, 1(1), 6-20.

Tyler, W. (1986). Principios básicos del currículo. (5 ed.). Buenos Aires: Editorial Troquel.

Villena, V. (2013). La evaluación de los aprendizajes y su incidencia en el rendimiento académico de los estudiantes del sexto, séptimo y octavo años del Centro de Educación Básica José Joaquín Olmedo de la parroquia de Ambatillo, cantón Ambato, provincia de Tungurahua, año lectivo 2010-2011. Recuperado desde http://repo. uta.edu.ec/handle/123456789/2579

Zárate, R. \& Socha, C. (2009). Estudio sobre las motivaciones de deserción estudiantil en la Universidad Industrial de Santander. Revista Prospectiva, (14), 1-25. 